\title{
ELECTROMAGNETIC AND CORPUSCULAR EMISSION FROM THE SOLAR FLARE OF 1991 JUNE 15: CONTINUOUS ACCELERATON OF RELATIVISTIC PARTICLES
}

\author{
L. G.KOCHAROV \\ St. Petersburg State Technical University, St. Petersburg, 195251, Russia
}

G. A. KOVALTSOV, G. E. KOCHAROV, E. I. CHUIKIN, I. G. USOSKIN A.F. Ioffe Physico-Technical Institute, St. Petersburg, 194021, Russia

M. A. SHEA, D. F. SMART

Space Physics Division, Geophysics Directorate, Phillips Laboratory, Hanscom AFB, Bedford, MA 01731-3010, U.S.A.

V. F. MELNIKOV, T. S. PODSTRIGACH

Radiophysical Research Institute, Nizhny Novgorod, 603600, Russia

T. P. ARMSTRONG

University of Kansas, Lawrence, KS 66045, U.S.A.

and

H. ZIRIN

Big Bear Solar Observatory, California Institute of Technology, Pasadena, CA 91125, U. S.A.

(Received 25 June, 1993)

\begin{abstract}
Data on X-, $\gamma$-ray, optical and radio emission from the 1991 June 15 solar flare are considered. We have calculated the spectrum of protons that produces $\gamma$-rays during the gradual phase of the flare. The primary proton spectrum can be described as a Bessel-function-type up to $0.8 \mathrm{GeV}$ and a power law with the spectral index $\approx 3$ from 0.8 up to $10 \mathrm{GeV}$ or above. We have also analyzed data on energetic particles near the Earth. Their spectrum differed from that of primary protons producing $\gamma$-ray line emission. In the gradual phase of the flare additional pulses of energy release occurred and the time profiles of cm-radio emission and $\gamma$-rays in the $0.8-10 \mathrm{MeV}$ energy band and above $50 \mathrm{MeV}$ coincided. A continuous and simultaneous stochastic acceleration of the protons and relativistic electrons at the gradual phase of the flare is considered as a natural explanation of the data.
\end{abstract}

\section{Introduction}

In the present paper we consider observations of the electromagnetic and particle emission from the powerful flare of 1991 June 15. During this flare, high-energy $\gamma$-ray emission up to $2 \mathrm{GeV}$ was observed by the GAMMA-1 telescope (Akimov et al., 1991; Leikov et al., 1993). This emission is a signature of processes of proton acceleration from $200 \mathrm{MeV}$ up to tens of GeV. Protons of such high energy have been detected previously in only a few ground level events (GLE) detected by the neutron monitor network (e.g., Smart and Shea, 1991). This flare was also observed by the GRO/COMPTEL device in the $0.8-30 \mathrm{MeV} \gamma$-ray band (McConnell et al., 1992,1993 ), which is mainly generated by $10-100 \mathrm{MeV}$ protons. Joint analysis of 
available electromagnetic and corpuscular emission data allows one to formulate the main properties of solar flare acceleration processes.

\section{Solar Observations}

The 1991 June 15 flare (3B/X12, N33 W69) occurred in the active region 6659, which was a source of a number of very powerful solar flares connected with strong solar proton events. The optical flare of 1991 June 15 began at 08:10 UT and reached maximum at 08:21 UT. According to $\mathrm{H} \alpha$ observations with the Large Coronagraph of the Astronomical Institute of Wrocław University (Rompolt, 1991) the flare displayed a very complicated structure with at least three ribbon-like features. While the flare covered about 50 arc sec, the kernel area between the ribbons is no more than $15 \operatorname{arcsec}$ or $10^{9} \mathrm{~cm}$ across. Surges and development of an eruptive loop as well as development of the post-flare loop system were also recorded. In Figure 1 we show the post-flare loops observed at Big Bear in the late stages of the flare. Top-to-bottom scale is $200 \mathrm{arc} \mathrm{sec}$, i.e., the size of the loops was about $10^{10} \mathrm{~cm}$.

According to GOES observations in the soft X-ray band (1-8, $0.5-4 \AA)$ the flare had an onset at 08:10 UT and a maximum at 08:21 UT simultaneous with the $\mathrm{H} \alpha$ flare and lasted for approximately 10 hours (Solar Geophysical Data, 1991). The soft X-ray intensity decreased a factor of ten from its maximum value during the next 1.5 hours. This flare was a typical long-duration X-ray flare according to the classification by Cane, McGuire, and von Rosenvinge (1986). Flares of such class are also called 'dynamic' flares (Pallavacini et al., 1977; Švestka, 1989). The temperature, $T$, and emission measure, $E M$, of the thermal source of soft X-ray emission can be deduced from observations in two energy bands (Crannell et al., 1978; Mätzler et al., 1978). Using the GOES observations we obtained temperature and emission measure: $T=10^{7} \mathrm{~K}$ and $E M=5 \times 10^{51} \mathrm{~cm}^{-3}$ during the impulsive phase at 08:15-08:20 UT. An hour later these values were $10^{7} \mathrm{~K}$ and $10^{51} \mathrm{~cm}^{-3}$. The flare evolution during the gradual phase in a $\log T$ vs $\log E M$-diagram is shown in Figure 2.

The time profiles of the flare radio emission observed at the radio-astronomical station 'Zimenki' (Nizhny Novgorod, Russia) at two frequencies are presented in Figure 3. The flare emission in centimeter and decimeter wavelength bands consisted of an impulsive phase at 08:12-08:25 UT and a subsequent IV(dm) and IV $(\mu)$ type burst lasting until about 10:00 UT. The type II meter wave radio burst was also observed from 08:16 UT and it lasted about $20 \mathrm{~min}$ (Solnechnye Dannye, 1991).

The impulsive phase of the flare in $\geq 2 \mathrm{MeV}$ photons was observed in a Pilot B scintillator anticoincidence shield of IMP 8 Charged Particles Measurement Experiment (CPME). Despite the fact that the CPME sensors are optimized for the detection of charged particles, sensitivity to uncharged particles, photons in this instance, is unavoidable. In the earliest stages of a solar flare particle event such as 


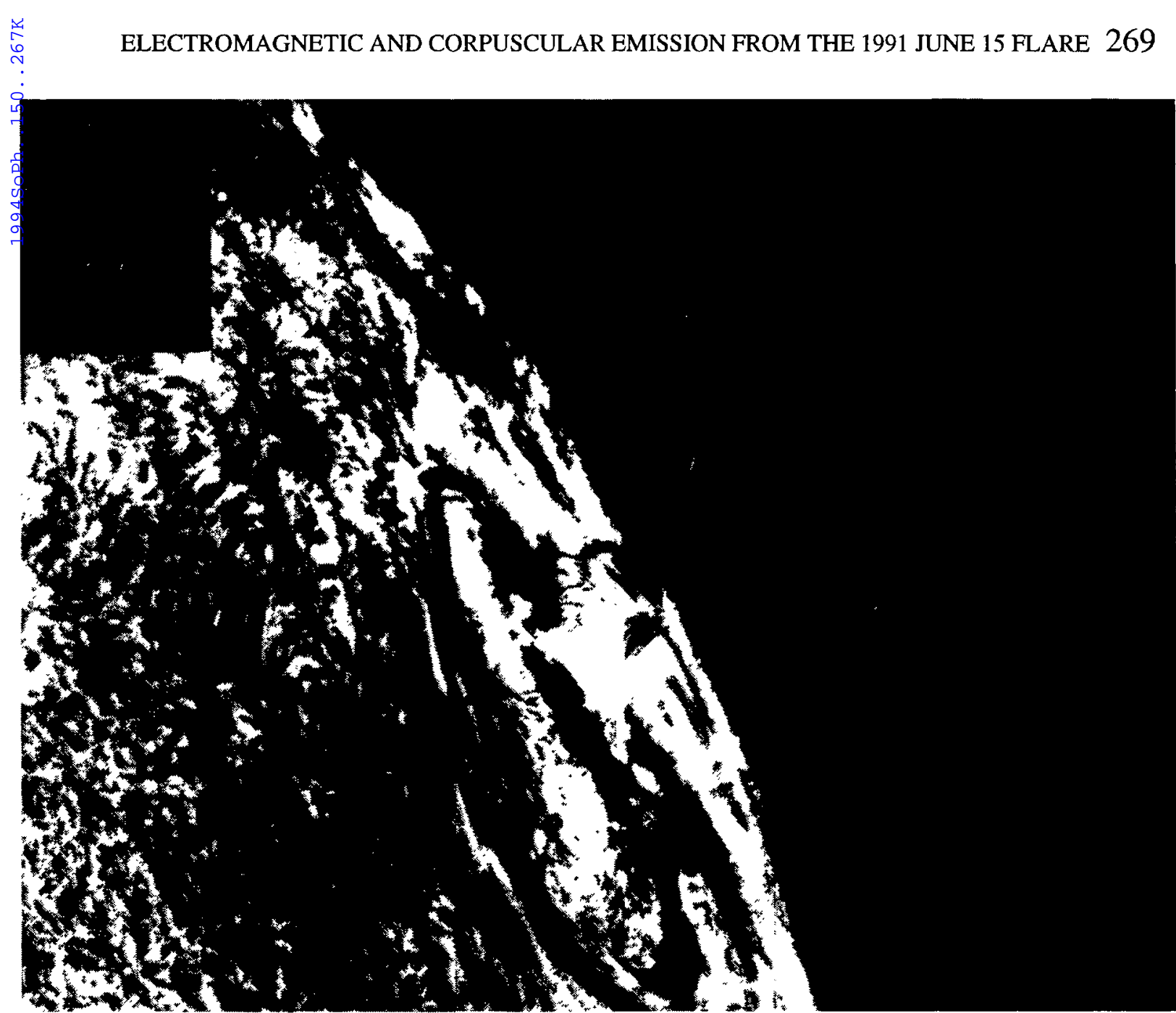

Fig. 1a. The sunspot group in H $\alpha$ just after sunrise for Big Bear at 15:44 UT of June 15. Post-flare loops are seen pouring into the spot area. Top-to-bottom scale, 200 arc sec.

the June 15,1991 event, the CPME response to solar X-rays is easily distmguished from the response due to charged particles. The count rate profile in the $\geq 2 \mathrm{MeV}$ CPME scintillator is very similar to the $9100 \mathrm{MHz}$ radio emission profile showing the occurrence of several sharp peaks at 08:15 UT and 08:17 UT and a decrease of the emission after 08:20 UT. The occurrence of multiple peaks in the hard X-ray onset just before 08:15 UT observed in this flare is typical of many large X-ray and particle emitting flares.

\section{Gamma-Ray Emission on 1991 June 15}

The time profiles of $\gamma$-ray emission during the June 15 flare are shown in Figure 3. From Figure 3 it is clear that observations of both GAMMA-1 (08:37-09:02 UT) and GRO/COMPTEL (08:58-09:37 UT) occurred after the impulsive phase of the flare (about 08:15 UT). The GAMMA-1 data show an exponential decay of high-energy $\gamma$-ray emission that lasted till about 08:50 UT followed by a plateau. The exponential time constant was about $9.8 \mathrm{~min}$. Figure 4 shows the count rate 


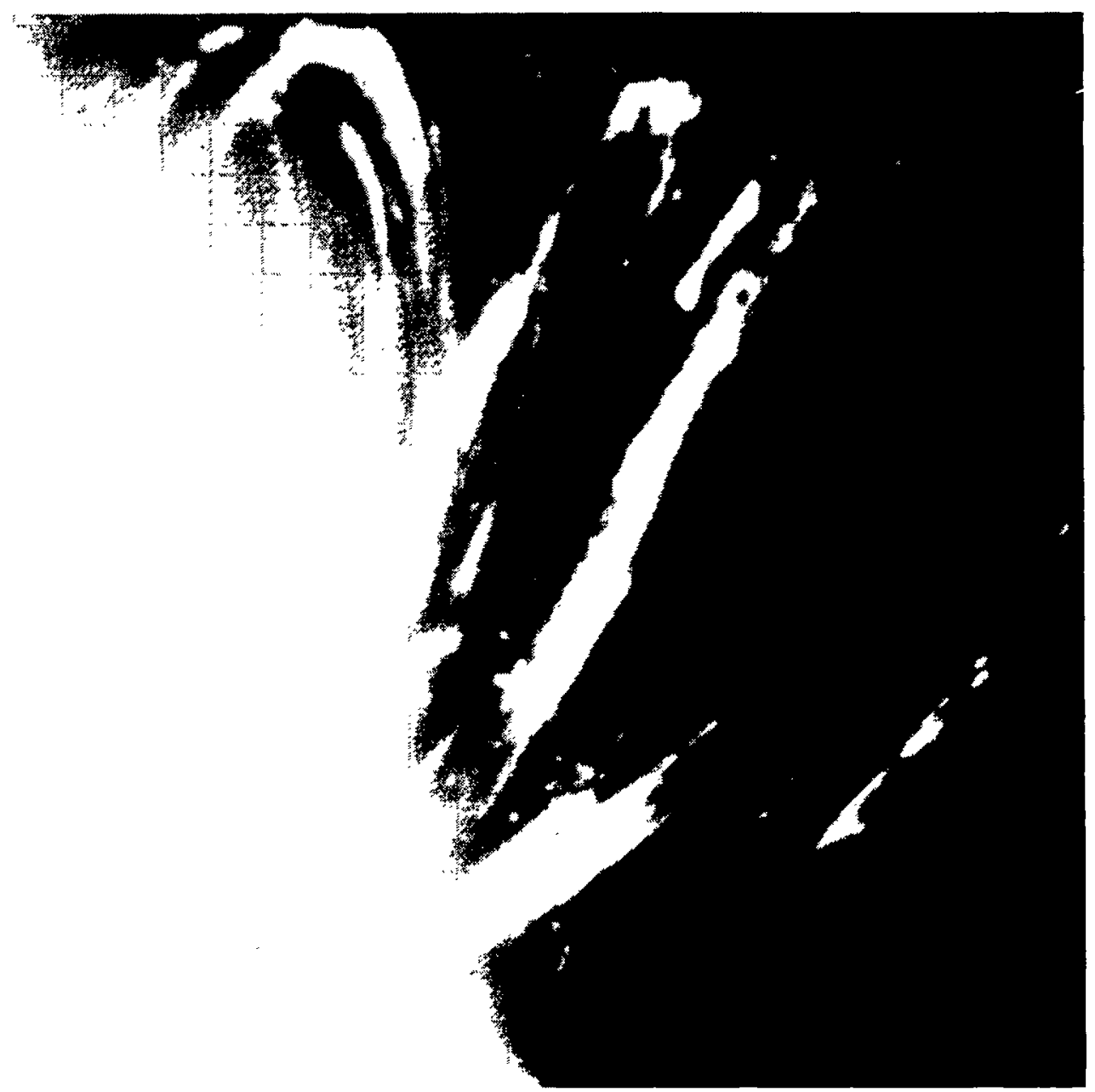

Fig. 1b. A highly overexposed frame at 17:12:53 UT (Big Bear observations) showing the faint post-flare loops continuing.

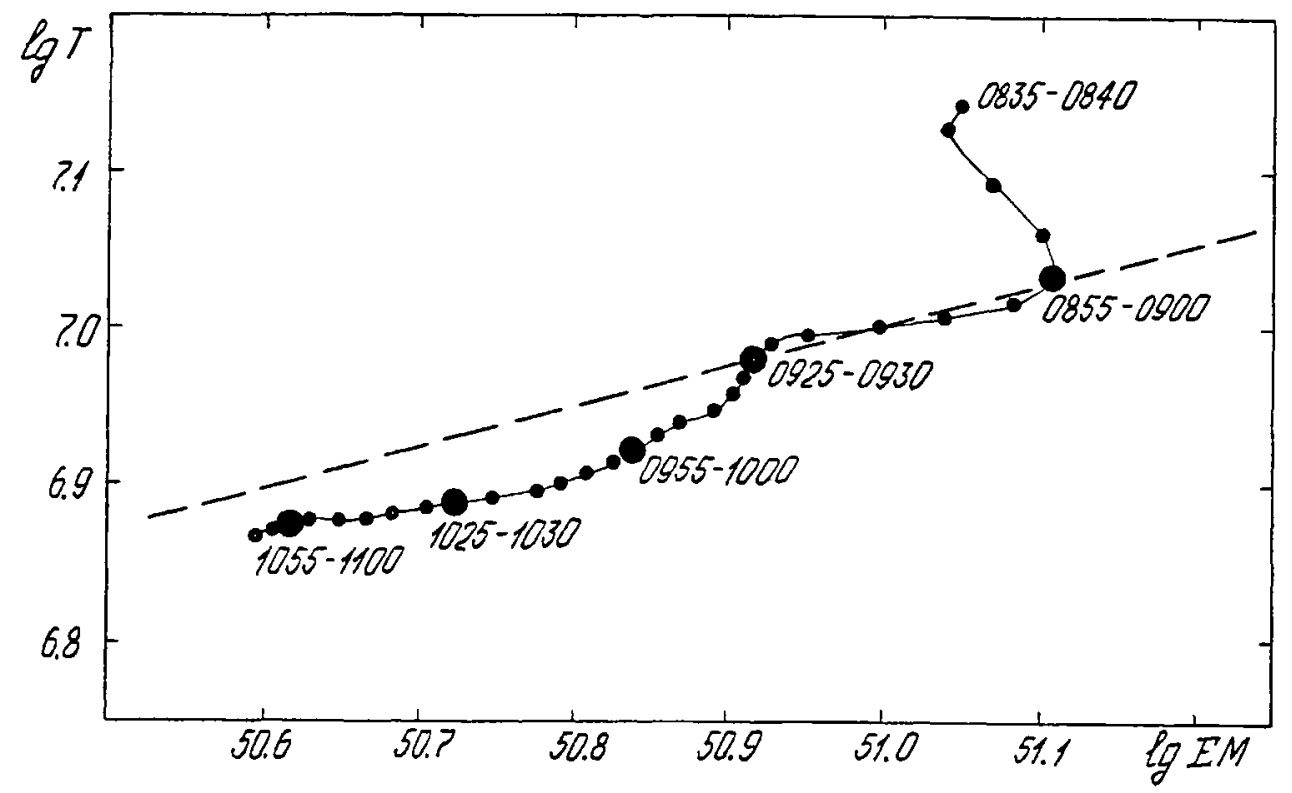

Fig. 2. The $(\log T)$ vs $(\log E M)$ diagram for the gradual phase of the flare. The temperature is taken in $\mathrm{K}$ and the emission measure in $\mathrm{cm}^{-3}$. Corresponding 5-min average time intervals (UT) are shown near the curve. The dashed line is the line of steady-state equilibria. 


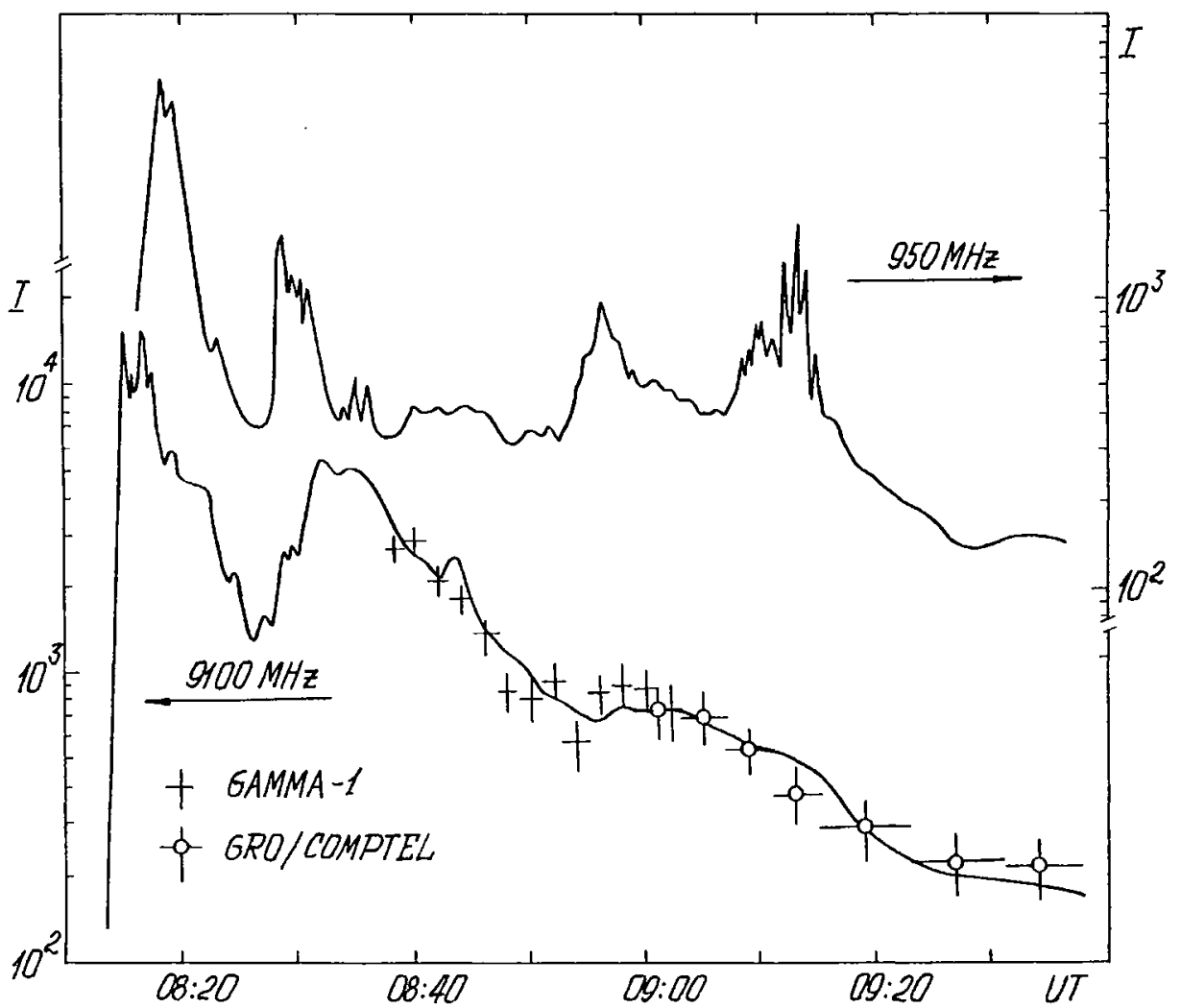

Fig. 3. Time profiles of radio- and $\gamma$-ray emission from the flare of 1991 June 15. GRO/COMPTEL: 0.8-10 MeV $\gamma$-rays (McConnell et al., 1993). GAMMA-1: above $50 \mathrm{MeV} \gamma$-rays. Radio emission intensity is taken in s.f.u., $\gamma$-rays in arbitrary units. The data are shown with background subtraction.

of GAMMA-1 in two energy ranges. From the comparison of $>50 \mathrm{MeV}$ and $>200 \mathrm{MeV}$ rates we can see that there was no significant variation of the energy spectrum up to 09:02 UT. After 09:02 UT the South-Atlantic Anomaly (SAA) affected the GAMMA-1 detectors and solar $\gamma$-rays were obscured. Hence we consider the GAMMA-1 count rate after 09:02 UT as the upper limit for solar $\gamma$-ray emission.

Akimov et al. (1991) proposed that the observed high-energy $\gamma$-ray emission originated from pion decay. We tested this hypothesis by use of our calculations of nuclear reactions in the solar atmosphere and GAMMA-1 original data on count rate. We used the thick target isotropic model of neutral pion generation in the solar atmosphere. The primary proton energy spectrum above $200 \mathrm{MeV}$ was modeled as a two-segment power-law cutoff at an energy $E_{m}$ as shown in Figure 5. Then we computed the expected GAMMA-1 count rates by means of the known response function (Akimov et al., 1988). The simulated device response was compared with the observed one by means of the $\chi^{2}(\nu)$ criterion (the number of energy intervals was $\nu+1$ ). First, a single power-law spectrum (in Figure 5, $S_{1}=S_{2}$ ) was analyzed, but it was impossible to obtain satisfactory agreement throughout the energy range. However, taking into account the high-energy range $\left(E_{\gamma}>250 \mathrm{MeV}\right)$ only, a good fit is obtained if the primary proton cutoff energy, $E_{m}$, is $\geq 10 \mathrm{GeV}$ (see Figure 5). 


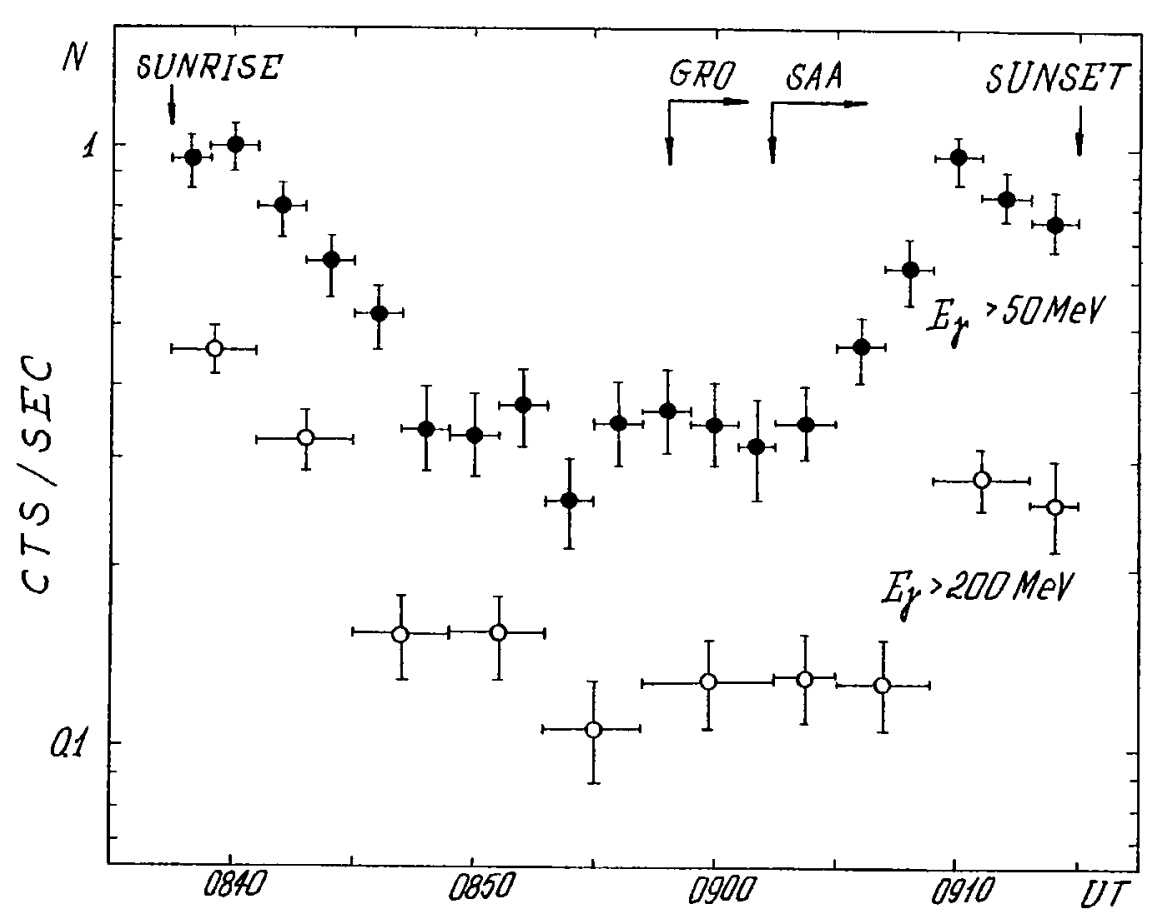

Fig. 4. Time history of GAMMA-1 count rate in two energy ranges. 'GRO' indicates the start time of the GRO/COMPTEL observations; 'SAA', the start time of the South-Atlantic impact. The data are shown without background subtraction. The background is 0.06 , and $0.015 \mathrm{cts} \mathrm{s}^{-1}$ for $>50 \mathrm{MeV}$ and $>200 \mathrm{MeV}$ energy range, respectively.

The best-fit proton power-law spectral index $S_{2}$ is equal to $3.5\left(\chi^{2}(4)=1.8\right)$. The $10 \%$ significance level (i.e.; $\chi^{2}(4)<7.8$ ) corresponds to proton spectral index $S_{2}$ from 2.9 up to 4.1 . The total number of protons accelerated above $1 \mathrm{GeV}$ for the 08:37-09:02 UT interval was about $10^{28}$ and was roughly independent of $S_{2}$. For the single power-law proton spectrum the calculated count rate in the $\gamma$-ray energy range below $250 \mathrm{MeV}$ is significantly less than that observed. Usually this fact is explained by additional relativistic electron bremsstrahlung (Kocharov et al., 1991; Ramaty et al., 1992; Mandzhavidze and Ramaty, 1992). On the other hand the observed excess of lower energy $\gamma$-ray emission can be explained using a steeper primary proton spectrum from $0.2 \mathrm{GeV}$ up to a certain $E_{0}$ (Kocharov et al., 1991). Proposing a double power-law shape of the spectrum ( $S_{1}>S_{2}$ in Figure 5) we compared the simulated and observed GAMMA-1 count rate in the whole energy range. We found a good agreement using the following proton spectrum parameters: $S_{2}=3, S_{1}=7, E_{0}=0.8 \mathrm{GeV}, E_{m} \geq 10 \mathrm{GeV}$, and $N_{p}(>1 \mathrm{GeV})=8 \times 10^{27}$. The corresponding $\gamma$-ray spectrum is shown in Figure 5. In order to visibly demonstrate the agreement between calculations and observational data we also plot the experimental points presented by Akimov et al. (1991).

Let us now consider the GRO/COMPTEL observations in the $0.8-30 \mathrm{MeV}$ band. Emission was visible up to $8 \mathrm{MeV}$ only. The neutron capture line at $2.2 \mathrm{MeV}$ and deexcitation nuclear lines were observed. This means that electron bremsstrahlung in 


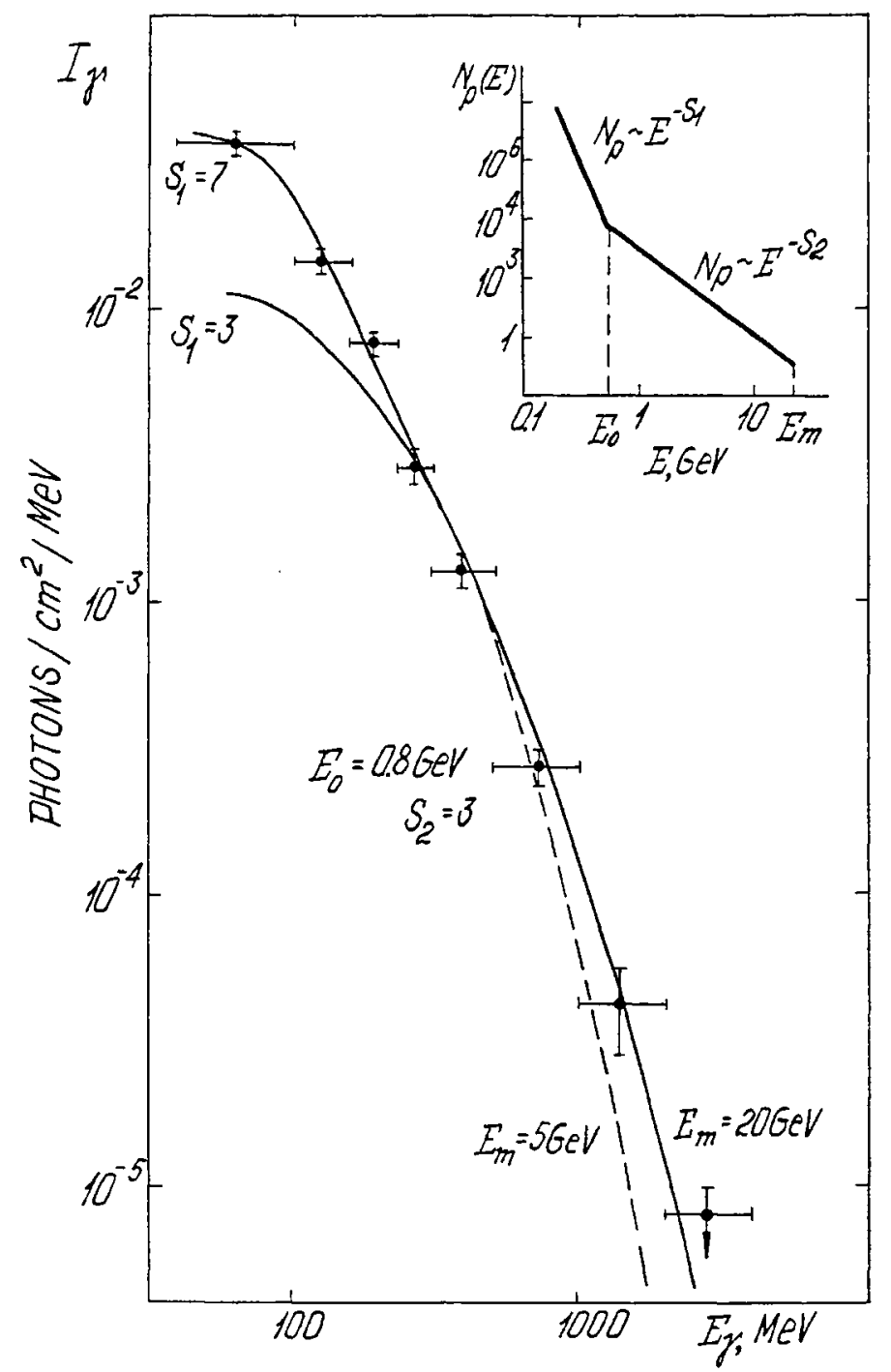

Fig. 5. Observed and calculated spectra of high-energy $\gamma$-ray emission (08:37-09:02 UT) and the primary proton spectrum used in the calculations. The dashed line demonstrates the discrepancy of calculated and observed spectra in the case of low cutoff energy, $E_{m}$.

the $1-8 \mathrm{MeV}$ energy range is small compared to nuclear $\gamma$-ray line emission which is produced by $10-100 \mathrm{MeV}$ protons. The obtained $\gamma$-ray line fluences were: $2.2 \mathrm{MeV}$ neutron capture line $-F(2.2)=11.1 \pm 1.5$ photons $\mathrm{cm}^{-2}$; carbon and oxygen lines in 4-7 MeV band $-F(4-7)=12.1 \pm 1.9$ photons $\mathrm{cm}^{-2}$ (McConnell et al., 1992). This gives the fluence ratio $F(2.2) / F(4-7)=0.92 \pm 0.21$. Using calculations by Hua and Lingenfelter (1987) for isotropic primary proton distribution, one can see that this ratio can be explained under the assumption of the proton spectrum being either of a Bessel-function-type (see, e.g., Forman, Ramaty, and Zweibel, 1986) with shape parameter $\alpha T=0.025-0.035$, or a power-law with spectral index $\approx 3$. The total number of protons $>30 \mathrm{MeV}$ for 08:58-09:37 UT interval was about $10^{32}$. Note that the determination of spectral parameters for primary protons is somewhat rough, in the case where only part of a flare is observed, because the 


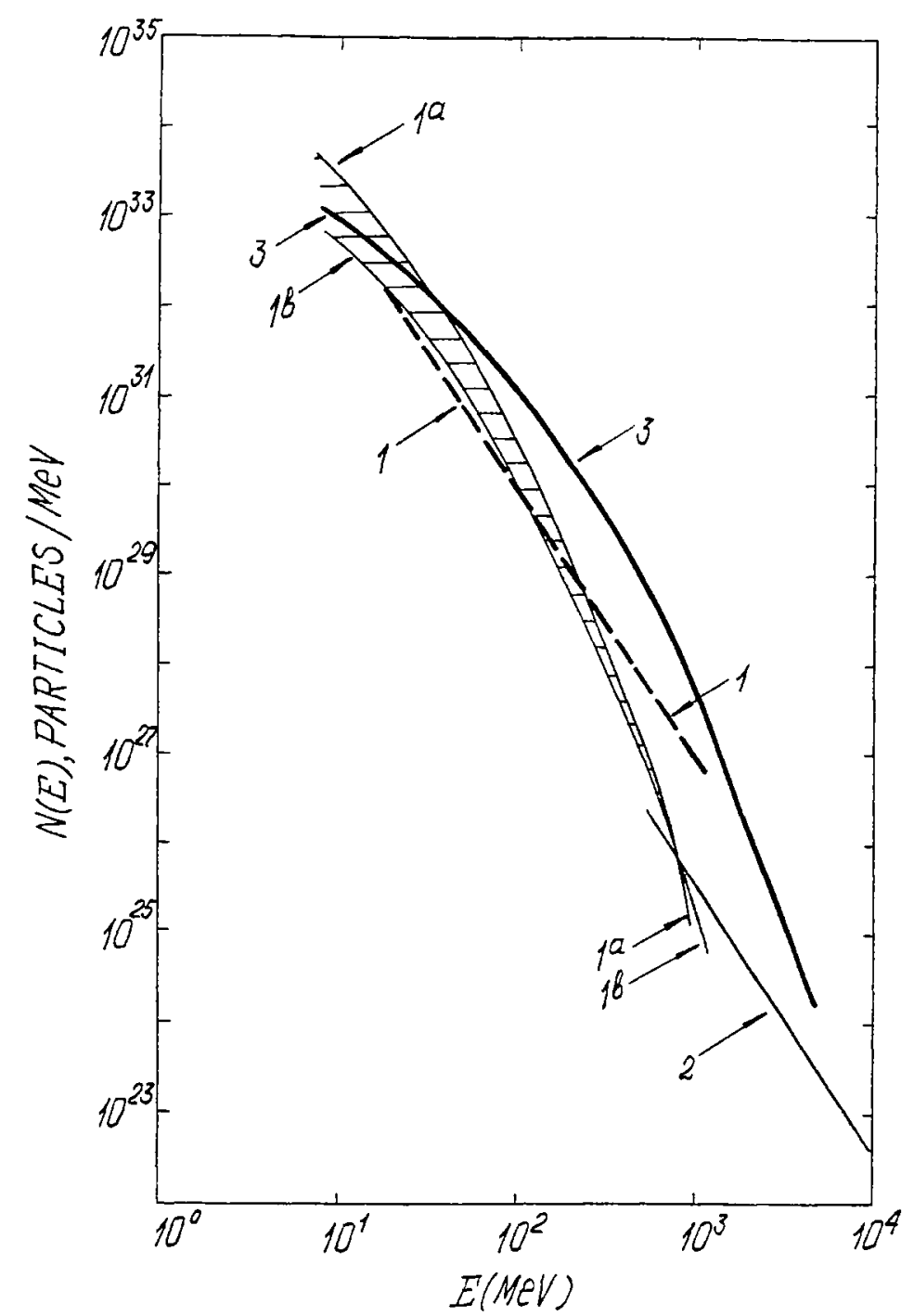

Fig. 6. Proton spectra at the Sun and in the interplanetary medium. $1 \mathrm{a}$ (or $1 \mathrm{~b}$ ) and 2 -primary proton spectrum in situ normalized to 08:37-09:02 UT interval; 1 - the power-law spectrum extrapolated from $10-100 \mathrm{MeV}$ range to $1 \mathrm{GeV}$ (for details see text); 3 - fit to the solar cosmic-ray data.

neutron capture line is delayed and lines of carbon and oxygen are not. However, it is not essential for the 1993 June 15 flare, because the duration of observations exceeds sufficiently the characteristic generation time of the $2.2 \mathrm{MeV}$ line (about 70 s, see, e.g., Trottet et al., 1993).

In the case where the power-law primary proton spectrum is in $10-100 \mathrm{MeV}$ range, one can extrapolate the spectrum to above $1 \mathrm{GeV}$ (see Curve 1 in Figure 6) and calculate the pion decay $\gamma$-ray emission. The GAMMA- 1 count rate obtained in this way is higher than the observed one by a factor of about 20 . On the other hand, the Bessel-function-type spectrum (Curves 1a, b) gives small enough calculated high-energy $\gamma$-ray emission to be in agreement with the observed one. It is essential that the Bessel-function-type spectrum steepen with energy (e.g., Miller, Ramaty, and Murphy, 1987) and become steep enough around $0.8 \mathrm{GeV}$ 


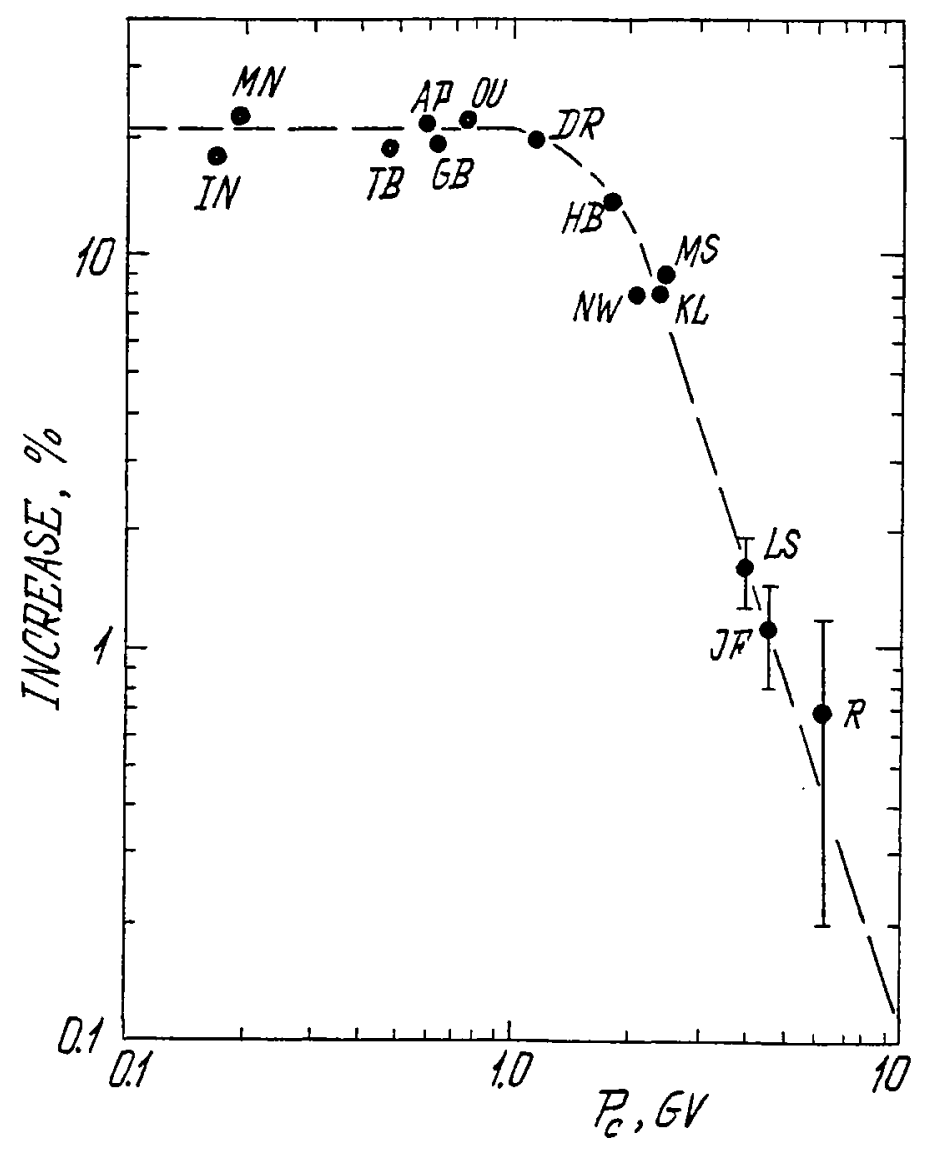

Fig. 7. Neutron monitor percent increases at the GLE maximum vs vertical cutoff rigidity. Neutron monitors: AP - Apatity, DR - Deep River, GB - Goose Bay, HB - Hobart, IN - Inuvik, JF - Jungfraujoch, KL - Kiel, LS - Lomnicky Štít, MS - Moscow, MN - Mawson, NW - Newark, OU - Oulu, $\mathrm{R}$ - Rome, TB - Tixie Bay.

in order to explain the excess of the $50-250 \mathrm{MeV} \gamma$-ray emission observed by GAMMA-1. Note that Curve 1a $(\alpha T=0.025)$ in Figure 6 gives a better fit to the GAMMA-1 data while Curve $1 \mathrm{~b}(\alpha T=0.03)$ demonstrates better coincidence with nuclear $\gamma$-ray line fluences detected by GRO/COMPTEL. Thus we conclude that the primary proton spectrum was approximately of a Bessel-function-type up to $0.8 \mathrm{GeV}$ situated between Curves $1 \mathrm{a}$ and $1 \mathrm{~b}$ in Figure 6 and a power law with spectral index $S_{2}=3$ (Curve 2) from 0.8 up to $10 \mathrm{GeV}$ or above during the whole GAMMA-1 and GRO/COMPTEL observation time. Both GAMMA-1 and GRO/COMPTEL observations can be explained under this proposition. In this case there is no necessity for additional relativistic electron bremsstrahlung in the $50-250 \mathrm{MeV}$ range. Note that under the proposition on anisotropic distribution of the primary protons even somewhat harder spectrum above $1 \mathrm{GeV}$ can be obtained (Kocharov et al., 1991). 


\section{Solar Cosmic Rays}

The flare of 1991 June 15 caused a ground-level solar cosmic-ray event which was observed by the world network of neutron monitors. In Figure 7 we plot the percent increase at the GLE maximum about 09:30 UT vs the vertical cutoff rigidity of the neutron monitors. All the increases are normalized by a sea-level monitor. All the neutron monitors with a geomagnetic cutoff rigidity, $P_{c}$, less than $5 \mathrm{GV}$ observed this event, independently of their longitude or local time. The increases for all the stations fit a smooth curve with only small deviations which is evidence for a low anisotropy of flare relativistic protons in the interplanetary medium at the GLE maximum. As seen from Figure 7 all the stations with $P_{c}<1 \mathrm{GV}$ observed the same count rate increase. This value of $P_{c}$ is the atmospheric threshold rigidity and is determined by the threshold of nuclear reactions of secondary nucleon generation in the Earth's atmosphere. That is, neutron monitors are not sensitive to solar cosmic rays below $1 \mathrm{GV}$. Note that a slight increase at the Rome monitor $\left(P_{c}=6.12 \mathrm{GV}\right)$ indicates that protons were accelerated up to rigidities greater than $6 \mathrm{GV}$.

We used the technique of GLE analysis developed by Shea and Smart (1982) and Shea et al. (1991) to determine the flux anisotropy and spectral characteristics of the 1991 June 15 event. This method of analysis seeks to reproduce, through the neutron monitor yield functions (Lockwood, Webber, and Hsieh, 1974; Debrunner and Flückiger, 1971) and the asymptotic directions of approach (Gall et al., 1982), the intensity/time profiles observed by the neutron monitors. The analysis method is based on the fact that the Earth's geomagnetic field acts as a 'filter' and only a restricted set of charged particle propagation directions in space are allowed at a specific point (cosmic-ray detector) on the surface of the Earth. The allowed orbits in the geomagnetic field establish the asymptotic directions of approach (or asymptotic cones of acceptance) which allow a determination of the high-energy particle directions in space prior to interactions with the Earth's magnetic field (McCracken, 1962). In order to carry out the analysis we had to know the directivity of the interplanetary magnetic field. Unfortunately there are no interplanetary magnetic field measurements for Earth-orbiting satellites during this event. The IMP-8 spacecraft was in the magnetospheric tail during the time of this event. However, since the energetic particle flux in space was determined to be only 'weakly anisotropic', this lack has only very small consequences in the event analysis. An assumption of a 'nominal' interplanetary magnetic field direction in the ecliptic plane is adequate for this circumstance.

We found that the high-energy particle spectrum at the maximum of the GLE may be fitted as $I=19.7 \times P^{-6}\left(\mathrm{GV} \text { ster } \mathrm{cm}^{2} \mathrm{~s}\right)^{-1}$, where $I$ is the particle flux averaged over all the directions and $P$ is the particle rigidity (GV). Note that because of real uncertainties the proton spectrum may be a bit harder but in any case not harder than $P^{-5.5}$. We found that the high-energy particle flux possessed only a 'mild' anisotropy during this event. In the decay of the GLE the 


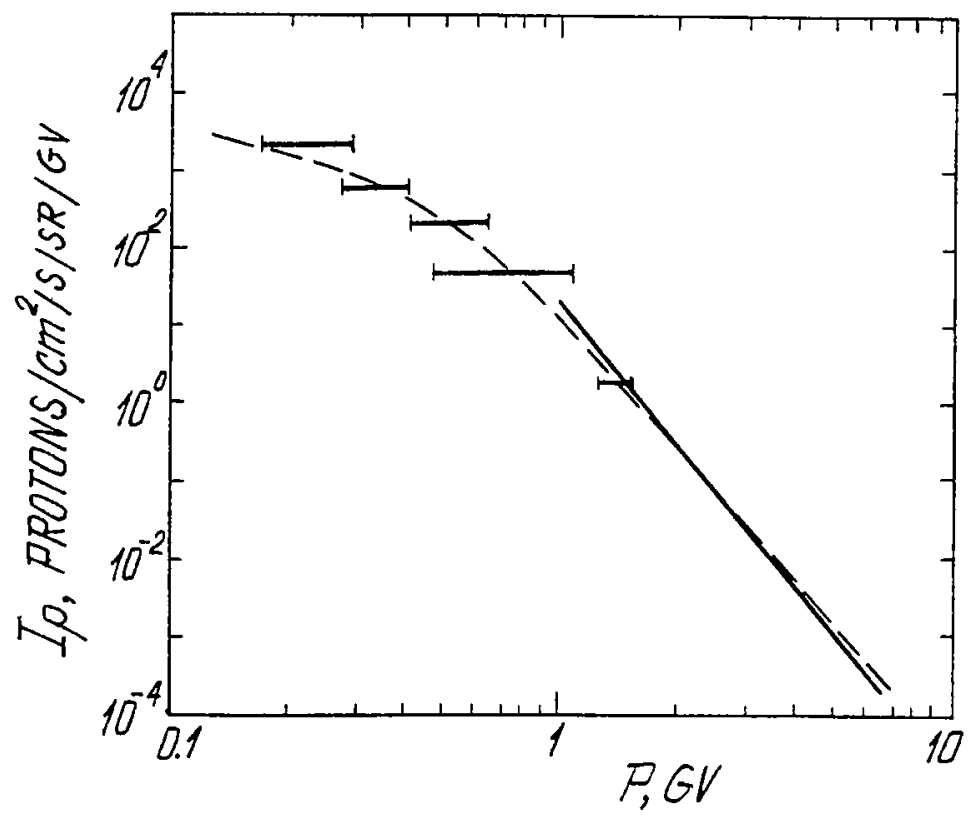

Fig. 8. The spectrum of protons at the Earth's orbit in the maximum of the event of 1991 June 15. The solid line indicates the spectrum derived from the analysis of neutron monitor data. The points indicate the satellite data. The dashed line is for the Bessel-function-type spectrum $(\alpha T=0.07)$.

anisotropy remains low and the spectrum softens a little. The relativistic proton spectrum obtained at the GLE maximum is shown in Figure 8. In the same figure we present the intensity at the solar cosmic-ray event maximum as observed by GOES-7 and GOES-6 satellites (Solar Geophysical Data, 1991). It is evidence that the spectra match each other. The spectrum of escaped protons obtained by neutron monitors and GOES data is fitted by Curve 3 in Figure 6. In order to evaluate the total number of particles in the interplanetary medium we use a simple isotropic diffusion model of particle propagation in the interplanetary magnetic field (Parker, 1963). Our estimate of the total number of particles escaped from the Sun are: $N_{p}(>39 \mathrm{MeV}) \approx 2.4 \times 10^{33}$ and $N_{p}(>1 \mathrm{GeV}) \approx 1.5 \times 10^{30}$. On the other hand, according to GAMMA-1 observations, only about $8 \times 10^{27}$ protons above $1 \mathrm{GeV}$ interacted at the Sun to generate the $\gamma$-rays. Thus in this energy range the number of escaping protons exceeded the number of interacting ones. At the same time, the total number of accelerated protons generating the $\gamma$-ray line emission and the number of escaping $\geq 10 \mathrm{MeV}$ protons were of the same order of magnitude (see Curves 1a, b and 3 in Figure 6). The observed spectrum of the escaped protons was of a Bessel function form and may be fitted by means of $\alpha T=0.065-0.07$, i.e., it was harder than the spectrum of the first population of the stopped protons $(\alpha T=0.025-0.03)$. That the spectrum of protons measured by neutron monitors and in interplanetary space can be harder than the proton spectrum deduced from $\gamma$-ray line emission was previously pointed out in a paper by Rieger et al. (1987).

Analyzing the dependence of the detection time of the first arriving particles on their energy one can obtain the time of their escape from the solar corona while 
a similar analysis for the time of the intensity maximum would yield the time of injection for the bulk of the particles (Ma Sung, Van Hollebeke, and McDonald, 1975; Reinhard and Wibberenz, 1974; Lockwood et al., 1987). The analysis is based on the fact that the interval between the arrival time of the first particles at Earth and the time of their injection from the corona is $L / v$, where $L$ is the distance traveled by the particles from the injection region to the Earth and $v$ is the speed of the particles. In Figure 9 we present onset times observed by the IMP- 8 satellite for four different energy channels. These data are plotted as $\beta^{-1}$ of each measurement interval where $\beta$ is the speed of the particles in units of the speed of light, $c$. We added $500 \mathrm{~s}$ (the time of light propagation from the Sun to the Earth) to any of the arrival times for convenience of comparison of the injection time with the time history of electromagnetic emission. The data may be fitted by a line with $c / L$ slope where $L \approx 1.2 \mathrm{AU}$. The value $L=1.2 \mathrm{AU}$ is reasonable for a western flare well connected with the Earth by the interplanetary magnetic field lines. One can see that protons with energies below $440 \mathrm{MeV}$ were first injected into interplanetary space at 08:25-08:33 UT (see the points of cross of the $c / L$ lines and the time axis in Figure 9). A similar analysis of GOES data gives a slightly earlier injection time. Thus, non-relativistic protons were injected soon after the impulsive phase of the flare. Neutron monitor data show that protons with energies above $1 \mathrm{GeV}$ were injected later at about 08:40 UT during the gradual phase of the flare. The analysis for the times of solar cosmic-ray event maximum showed that the bulk of particles of all energies was injected into interplanetary space after 09:00 UT.

\section{Discussion}

According to 'Zimenki' station observations, the flare of 1991 June 15 was characterized by a continuous generation of radio emission which had a complicated multi-impulsive structure. It is seen from Figure 3 that the first pulse of centimeter radio emission $(9100 \mathrm{MHz})$ at $08: 15-08: 25$ UT corresponds to the impulsive phase of the solar flare. It is followed by two increases corresponding to the gradual phase. Pulse increase intervals in the centimeter band correspond to maxima in the decimeter band (950 MHz) at about 08:30 UT and 08:56 UT. The decimeter emission may be caused by plasma radio emission and follows continuous multi-impulsive energy release processes. The centimeter radio emission is usually interpreted as synchrotron emission of $\mathrm{MeV}$ electrons accelerated as a result of energy release. During the impulsive phase $\geq 2 \mathrm{MeV}$ electrons were also observed by their bremsstrahlung resulting in the CPME count rate increase (Section 2).

According to Jakimiec et al. (1986), the $(\log T, \log E M)$-diagram allows one to investigate quantitatively the behavior of the heating function in a flare. It is seen from the comparison of results of calculations by Pallavicini et al. (1983) with the empirical Figure 2 that the heating operates during the gradual phase, allowing the flare to reach a quasi-steady state at about 08:55 UT. The flare evolution at 08:55-09:20 UT may be explained as due to cooling along the line of steady-state 


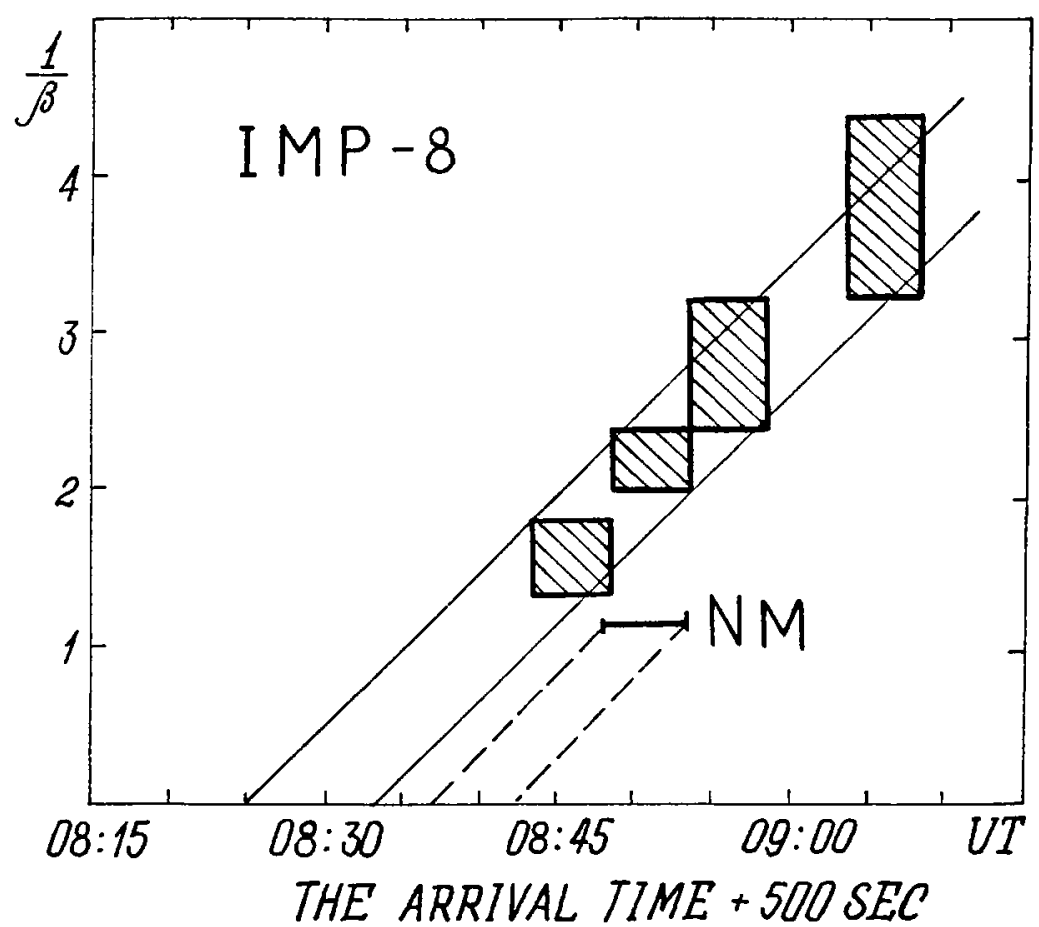

Fig. 9. The times of the first arrival of particles vs $\beta^{-1}$. The shaded box indicates the spread in the speed of the particles and the uncertainty in the determination of the first arrival time. ' $N M$ ' is for the arrival of relativistic protons according to neutron monitor data. The slope of lines is $c / L$.

equilibria at a gradual decrease in the heating. Thus we consider the decimeter radio and soft X-ray emissions as evidence of continuous energy release after the impulsive phase of the flare. Švestka (1989) discussed observations and physical processes in the gradual phase of dynamic flares and concluded that in these flares energy continues to be released for a long time after the end of the impulsive phase. Evidently this delayed energy release occurs in a giant magnetic structure extending to at least $2 \times 10^{10} \mathrm{~cm}$ altitude (Kai et al., 1986).

As is seen from a comparison of Figures 3 and 9, the onset of non-relativistic proton injection into the interplanetary medium followed the first (impulsive phase) increase in the $\mathrm{cm}$-band and coincided with the type II radio burst. The onset of the second peak in the cm-band (08:30 UT), arising in the gradual phase of the flare, was followed by the relativistic proton injection. At the same time, highenergy $\gamma$-ray emission generated by the interacting relativistic protons in the solar atmosphere was detected. The number of escaping protons was more than or about the number of protons which produced the nuclear $\gamma$-ray lines. This is typical for gradual (long-duration) flares (Kocharov et al., 1984; Kocharov and Kovaltsov, 1986).

There are two possibilities to explain the long-duration $\gamma$-ray emission. The first is continuous acceleration of particles during the solar flare. This explanation would be in accordance with the above interpretation of soft X-ray and radio emission. The second possibility is acceleration during the impulsive phase followed by 
trapping of energetic particles in a magnetic loop (Mandzhavidze and Ramaty, 1992). In Figure 3 we show overlapping time profiles of $\mathrm{cm}$ radio emission and $\gamma$ ray radiation observed by GAMMA- 1 and GRO. One can see that the time profiles in the centimeter band and of $\gamma$-ray radiation in nuclear lines and resulting from pion-decay coincide. Energy losses of particles producing these kinds of emission differ significantly from each other and so the observed temporal behavior of those kinds of emission cannot be explained by primary particle deceleration. In trapping models, however, the temporal behavior of the secondary emission is mainly determined by precipitation of particles due to scattering on MHD waves (Gueglenko et al., 1990). The exponential decay of emission is typical for trapping models but, in the general case, decay times are different for different kinds of emission because of the dependence of scattering time on particle gyroradius. Thus, in order to explain the observations on the base of the trapping model it is necessary to propose the action of some special process which scatters all kinds of particles with the same efficiency. Under this ad hoc assumption it seems to be possible to propose that acceleration occurs only during the impulsive phase of the flare. In this case one has to extrapolate the total number of interacting relativistic protons back to the impulsive phase, using an exponential law with decay time of 9.8 min obtained from GAMMA-1 observation. Accordingly, during the impulsive phase, these protons would produce the secondary high-energy neutron flux which could surely be detected by the Alma Ata neutron monitor. The geomagnetic cutoff rigidity of the Alma Ata neutron monitor, $P_{c}$, is $6.7 \mathrm{GeV}$, which decreases significantly its sensitivity to solar protons, while the small solar zenith angle and high altitude of the monitor give a good possibility of detecting high-energy solar neutrons generated by interacting protons at the Sun (cf. Kovaltsov et al., 1993). However, the time history of the Alma Ata neutron monitor count rate demonstrates no sharp increase during the impulsive phase. As a result, the estimated number of primary protons at the Sun during the impulsive phase did not exceed their quantity during the gradual phase. Thus the trapping model should be abandoned in favor of (continuous) acceleration of relativistic protons after the impulsive phase of the flare.

A shock wave which produces type II radio emission is a traditional candidate for the second stage acceleration. The meter-wave type II radio emission had its onset at 08:16 UT in the impulsive phase and a duration of about $20 \mathrm{~min}$. Thus one can consider a third possibility by proposing that relativistic protons were accelerated just before the GAMMA-1 observation onset. However, in this case all theoretical problems concerning the trapping arise again. Besides, one has to explain the delayed escape of relativistic protons detected at $1 \mathrm{AU}$. That is why we conclude that the first possibility, which proposes a continuous acceleration of relativistic particles during the gradual phase, is the best. It gives a natural explanation of the temporal behavior of microwave and $\gamma$-ray emission during the 08:37-09:37 UT interval, i.e., tens of minutes after the impulsive phase. It is also in accordance with the fact that protons above $1 \mathrm{GeV}$ observed near the Earth started to escape 
from the Sun about $10 \mathrm{~min}$ later than non-relativistic protons originating from the impulsive phase (Section 4). Thus we consider the emission observed during the gradual phase to be the result of a continuous and simultaneous acceleration of protons and electrons in a wide range of energy. Stochastic acceleration models are the most suitable for the explanation with the data in hand. It may be acceleration by an ensemble of shock waves (Bykov and Toptyghin, 1981) excited due to multiimpulsive energy release proposed in the stochastic models of solar flares (Vlahos, 1989). The two-component interacting proton spectrum obtained above (soft Bessel function type and hard power law) may originate from a special structure of the accelerated region. A more energetic 'kernel' (or 'kernels') of the acceleration region may be a source of the hard power-law tail above $1 \mathrm{GeV}$ and the more quiet 'halo' may be a source of protons with the Bessel-function-type spectrum.

As was shown above, the spectrum of escaping protons was approximately of the Bessel function type. However, the spectrum parameter, $\alpha T$ for the escaping protons differed significantly from the interacting proton parameter. Having in mind that the total number of $>10 \mathrm{MeV}$ protons in interacting and escaped populations were of about the same order of magnitude, we can propose that the escaping protons originated from the same population of accelerated particles as the interacting ones, and some re-acceleration process is responsible for the hardening of the spectrum of protons before their escape. Apparently the (re-)acceleration region of escaping protons was situated higher in the solar corona and was of greater spatial scale.

\section{Concluding Remarks}

Recently the $\gamma$-radiation of the 1991 June 11 solar flare was reported (Trottet et al., 1993; Kanbach et al., 1993). That flare took place in the same active region as that of the June 15 flare. It is seen from the June 11 observations that the time intervals and spatial scales are three times those for the June 15 event. The first time scale of about a minute characterized the impulsive phase. Then a $25-\mathrm{min}$ $e$-folding time was observed during two hours. Finally, a 255-min $e$-folding time of high-energy $\gamma$-ray emission was seen during at least 6 hours. One can propose that three spatial scales correspond to these three time scales, e.g., $\sim 10^{9} \mathrm{~cm}$, $\sim 10^{10} \mathrm{~cm}$, and $\sim 10^{11} \mathrm{~cm}$. In the case of the June 15 flare a spatial scale of about $10^{9} \mathrm{~cm}$ corresponds to the scale of the kernel area in optical emission, while $10^{10} \mathrm{~cm}$ is the scale of the post-flare loop system (Section 2). For the June 15 flare we have $\gamma$-ray observations for the second time interval considered above as an interval of extended energy release and acceleration. Note that high-energy $\gamma$-ray spectra observed in the second interval of both flares were similar and so a similar interpretation of these spectra may be possible. It was reported (Akimov et al., 1991; Leikov et al., 1993) that high-energy $\gamma$-ray emission on June 15 was seen at the next GAMMA-1 orbit too, i.e., two hours after the impulsive phase. It is possible to propose that this emission belongs to the third time interval considered 
as an interval of trapping of accelerated protons. In the case of the June 15 flare, we have insufficient information to verify this hypothesis but it is possible to propose that this emission was due to the trappping of relativistic protons in the giant magnetic arch of $\sim 10^{11} \mathrm{~cm}$ size.

Thus, during the impulsive phase of the 1991 June 15 flare, non-relativistic protons detected near the Earth and $\approx 2 \mathrm{MeV}$ electrons were accelerated. Later, in the gradual phase, additional pulses of energy release occurred and continuous and simultaneous acceleration of relativistic protons and electrons took place. During the gradual phase, the spectrum of interacting protons consisted of two components: the first one dominating at non-relativistic energies had the Bessel-function-type spectrum, while the spectrum of the second component was a hard power law up to $10 \mathrm{GeV}$ or more. At the same time, relativistic protons with energies up to $5 \mathrm{GeV}$ were injected into the interplanetary medium. However, their spectrum differed from the one of the primary protons interacting at the Sun and producing $\gamma$-ray line emission. These features of the spectra may be considered as a signature of a complicated structure of the proton acceleration region.

\section{Acknowledgements}

It is a pleasure to acknowledge helpful discussions on these topics with Prof. J. A. Simpson and Prof. V. Petrosian. We are grateful to Dr E. V. Vashenyuk for providing material discussed here. GOES X-ray information used in this study was provided by WDC-A for Solar Terrestrial Physics, NOAA E/GC2, 325 Broadway, Boulder, Colorado 80303, U.S.A. The research at Big Bear Solar Observatory and in St. Petersburg was supported by NSF under Grant ATM-9122023 under Grant NAGW-1972.

\section{References}

Akimov, V. V., Balebanov, V. M., Belaousov, A. S. et al.: 1988, Space Sci. Rev. 49, 125.

Akimov, V. V., Afanassyev, V. G., Belaousov, A. S. et al.: 1991, Proc. 22nd Int. Cosmic Ray Conf., Dublin 3, 73.

Bykov, A. M. and Toptyghin, I. N.: 1981, Izv. AN SSSR, Ser. Fiz. 45, 474.

Cane, H. V., McGuire, R. E., and von Rosenvinge, T. T.: 1986, Astrophys. J. 301, 448.

Crannell, C. J., Frost, K. J., Matzler, C., Ohki, K., and Saba, J. L.: 1978, Astrophys. J. 223, 620.

Debrunner, H. and Flückiger, E.: 1971, Proc. 12th Int. Cosmic Ray Conf., Hobart 3, 911.

Forman, M. A., Ramaty, R., and Zweibel, E. G.: 1986, in P. A. Sturrock (ed.), Physics of the Sun, Vol. 2, D. Reidel Publ. Co., Dordrecht, Holland, p. 249.

Gall, R., Orozco, A., Marin, C., Hurtado, A., and Vidargas, G.: 1982, Tech. Rep., Instituto de Geofísica, Universidad Nacional Autonoma de Mexico.

Gueglenko, V. G., Kocharov, G. E., Kocharov, L. G., Kovaltsov, G. A., and Mandzhavidze, N. Z.: 1990, Solar Phys. 125, 91.

Hua, X.-M. and Lingenfelter, R. E.: 1987, Solar Phys. 107, 351.

Jakimiec, J., Sylwester, B., Sylwester, J., Lemen, J. R., Mewe, R., Bentlley, R. D., Peres, G., Serio, S., and Schrijver, J.: 1986, in V. E. Stepanov and V. N. Obridko (eds.), Solar Maximum Analysis, VNU Science Press, Utrecht, p. 91. 
Kai, K., Nakajima, H., Kosugi, T., Stewart, R. T., Nelson, G. J., and Kane, S. R.: 1986, Solar Phys. $105,383$.

Kanbach, G., Bertsch, D. L., Fichtel, C. E., Hartman, R. C., Hunter, S. D., Kniffen, D. A., Kwok, P. W., Lin, Y. C., Mattox, J. R., Mayer-Hasselwander, H. A., Michelson, P. F., von Montigny, C., Nolan, P. L., Pinkau, K., Rothermel, H., Schneid, E., Sommer, M., Sreekumar, P., and Thompson, D. J.: 1993, Astron. Astrophys. Suppl. Ser. 97, 349.

Kocharov, G. E., Kovaltsov, G. A., Kocharov, L. G., and Semukhin, P. E.: 1984, in G. E. Kocharov (ed.), Energetic Particles and Photons from Solar Flares, Phys.-Tech. Inst. Publ., Leningrad, USSR, p. 46 (in Russian).

Kocharov, L. G. and Kovaltsov, G. A.: 1986, in Proc. of Joint Varenna-Abastumani Int. School and Workshop on 'Plasma Astrophysics', ESA SP-51, Paris, France, p. 101.

Kocharov, L. G., Kovaltsov, G. A., Guglenko, V. G., Kartavyh, Yu. Yu., Kocharov, G. E., and Usoskin, I. G.: 1991, in G. E. Kocharov (ed.), Nuclear Astrophysics, Phys.-Tech. Inst. Publ., St. Petersburg, Russia, p. 5.

Kovaltsov, G. A., Efimov, Yu. E., and Kocharov, L. G.: 1993, Solar Phys. 144, 195.

Liekov, N. G., Akimov, V. V., Volzhenskaya, V. A., Kalinkin, L. F., Nesterov, V. E., Galper, A. M., Zemskov, V. M., Oserov, Y. U., Topchiev, N. P., Fradkin, M. I., Tchuikin, E. I., Tugaenko, V. Y., Gros, M., Grenier, I., Bazer-Bachi, A. R., Lavigne, J. M., and Olive, J. F.: 1993, Astron. Astrophys. Suppl. Ser. 97, 345.

Lockwood, J. A., Webber, W. R., and Hsieh, L.: 1974, J. Geophys. Res. 79, 4149.

Lockwood, J. A., Debrunner, H., McGuire, R. E., and Quenby, J. J.: 1987, Proc. 20th Int. Cosmic Ray Conf., Moscow 3, 17.

Mandzhavidze, N. and Ramaty, R.: 1992, Astrophys. J. 396, L111.

Ma Sung, L. S., Van Hollebeke, M. A., and McDonald, F. B.: 1975, Proc. 14th Int. Cosmic Ray Conf., München 5, 1767.

Mätzler, C., Bai, T., Crannel, C. J., and Frost, K. J.: 1978, Astrophys. J. 223, 1058.

McConnell, M., Connors, A., Forrest, D. et al.: 1992, Report at 'Recent Advance in High Energy Astronomy', Toulouse, France.

McConnell, M., Bennett, K., Forrest, D., Hanlon, L., Ryan, J., Schönfelder, V., Swanenburg, B. N., and Winkler, C.: 1993, Adv. Space Res. 13(9), 245.

McCracken, K. G.: 1962, J. Geophys. Res. 67, 423.

Miller, J. A., Ramaty, R., and Murphy, R. J.: 1987, Proc. 20th Int. Cosmic Ray Conf., Moscow 3, 33.

Pallavicini, R., Serio, S., and Vaiana, G. S.: 1977, Astrophys. J. 216, 108.

Pallavicini, R., Peres, G., Serio, S., Vaiana, G., Acton, L., Leibacher, J., and Rosner, R.: 1983, Astrophys. J. 270, 270.

Parker, E. N.: 1963, Interplanetary Dynamical Processes, Interscience, New York.

Ramaty, R., Hua, X. M., Kozlovsky, B., Lingenfelter, R. E., and Mandzhavidze, N.: 1992, Proc. Compton Observatory Science Workshop, NASA CP 3137, p. 480.

Reinhard, R. and Wibberenz, G.: 1974, Solar Phys. 36, 473.

Rieger, E., Forrest, D. J., Bazilevskaya, G., Chupp, E. L., Kanbach, G., Reppin, C., and Share, G. H.: 1987, Proc. 20th Int. Cosmic Ray Conf., Moscow 3, 65.

Rompolt, B.: 1991, in FLARES 22/MAX '91 Summary of Campaigns, Alan Kiplinger, Max '91 Coordinator.

Shea, M. D. and Smart, D. F.: 1982, Space Sci. Rev. 32, 251.

Shea, M. A., Smart, D. F., Wilson, M. D., and Flückiger, E. O.: 1991, Geophys. Res. Letters 18, 209.

Smart, D. F. and Shea, M. A.: 1991, Proc. 22nd Int. Cosmic Ray Conf., Dublin 3, 101.

Solar Geophysical Data: 1991, 568, Pt. II.

Solnechnye Dannye: 1991, No. 6 (in Russian).

Švestka, Z.: 1989, Solar Phys. 121, 399.

Trottet, G., Vilmer, N., Barat, C., Dezalay, J. P., Talon, R., Sunaev, R., Kuznetsov, A., and Terekhov, O.: 1993, Astron. Astrophys. Suppl. Series 97, 337.

Vlahos, L.: 1989, Solar Phys. 121, 431. 